\title{
Universal Amplitude Ratios in the Ising Model in Three Dimensions
}

\author{
A. Gordillo-Guerrero ${ }^{1,2}$, R. Kenna ${ }^{3}$, and J.J. Ruiz-Lorenzo ${ }^{4,2}$ \\ ${ }^{1}$ Departamento de Ingeniería Eléctrica, Electrónica y Automática, \\ Universidad de Extremadura, Avda Universidad s/n, \\ Cáceres, 10071, Spain. \\ ${ }^{2}$ Instituto de Biocomputación and \\ Física de Sistemas Complejos (BIFI), \\ Zaragoza, 50009, Spain. \\ 3 Applied Mathematics Research Centre, \\ Coventry University, Coventry, CV1 5FB, England \\ ${ }^{4}$ Departamento de Física, \\ Universidad de Extremadura, Avda Elvas s/n, \\ Badajoz, 06071, Spain.
}

May 28, 2018

\begin{abstract}
We use a high-precision Monte Carlo simulation to determine the universal specific-heat amplitude ratio $A_{+} / A_{-}$in the three-dimensional Ising model via the impact angle $\phi$ of complex temperature zeros. We also measure the correlationlength critical exponent $\nu$ from finite-size scaling, and the specific-heat exponent $\alpha$ through hyperscaling. Extrapolations to the thermodynamic limit yield $\phi=$ $59.2(1.0)^{\circ}, A_{+} / A_{-}=0.56(3), \nu=0.63048(32)$ and $\alpha=0.1086(10)$. These results are compatible with some previous estimates from a variety of sources and rule out recently conjectured exact values.
\end{abstract}




\section{Introduction}

Although it is one of the most investigated models of statistical mechanics, the stillunsolved Ising model in three dimensions has recently again come under the spotlight [1, 2, 3, 4, 5, 6, 7, 8, 9, 10, 11, 12, This is because of (i) controversial claims as to the exact values of the critical exponents from analytical means and (ii) advances in algorithmic approaches which have allowed for greater numerical precision.

On the analytic side, two relatively recent papers claim to have found the exact exponents of the 3D Ising model [1, 2]. They claim rational values for the critical exponents, including $\alpha=0$ and $\nu=2 / 3[3]$. A speculative formula embracing the results for the magnetic exponent $\delta$ was subsequently given in Ref. [4] and the conjectured values for critical parameters in three dimensions were used in Ref. [5]. Despite criticism in Refs. 6, 17, experimental support for these critical exponents has been claimed in Ref. 8]. Further theoretical arguments in favour of these exponents are given in Ref. [9] (see also Ref.[10]). Refs. [1, 2, 3] also advocate the existence of a multiplicative logarithmic correction in the critical behaviour of the specific heat in the model.

Given the intriguing coincidence of the claimed values of the critical exponents in Ref.[1] and Ref.[2], and their connection with the Rosengren conjecture [13], precise measurement of $\alpha$ and $\nu$ are of renewed interest. Precision is important here, not least because if any disagreement with RG is found, this will have serious implications for one of the foundation stones of theoretical physics. This is also why the claims of Refs. [1, 2] caused so much controversy - they are not in agreement with precise, though non-exact, results from other methods. Moreover, while the values of the critical exponents claimed in Refs. [1, 2] obey the standard scaling relations, a logarithmic term in the specific heat would violate the scaling relations for logarithmic corrections unless certain conditions hold for the for the locus of Fisher zeros [14].

Although critical exponents have more commonly been used, amplitude ratios often offer a more discerning way to identify universality classes [15]. These have again recently received increased attention in the case of the of the $3 \mathrm{D}$ Ising model because improved numerical techniques now permit more stringent investigations. The most recent Monte Carlo simulations have yielded the estimates $A_{+} / A_{-}=0.532(7)$ [1] and $A_{+} / A_{-}=0.536(2)[12$ and these compare well with previous estimates from a variety of approaches in Table 1. The first entry in the table is conspicuous in that (i) it is significantly different to the other estimates and (ii) it comes from measuring the angle of impact of complex-temperature Fisher zeros onto the critical point, instead of through the more direct approaches employed by the other references cited. Therefore we considered it worthwhile revisiting this method, making use of the greater computational power available today, in an effort to determine whether the inconsistency is indicative of a deeper problem or whether it is simply down to limited numerics. For these reasons, our analysis focuses on the scaling and impact angles.

Our detailed numerical analysis upholds previous estimates for the critical exponents

coming from a variety of approaches. It also brings specific-heat amplitude-ratio estimates from the impact-angle approach into line with other estimates and rules out the recent conjectures for exact exponents. 


\section{Model and observables}

The partition function of the pure 3D Ising model on a lattice of extent $L$ without an applied magnetic field and at an inverse temperature $\beta=1 / T$ is

$$
Z_{L}(\beta)=\sum_{\left\{\sigma_{i}\right\}} \exp \left(\beta \sum_{\langle i, j\rangle} \sigma_{i} \sigma_{j}\right),
$$

where the sum over configurations $\left\{\sigma_{i}\right\}$ is taken over Ising spins, $\sigma_{i} \in\{ \pm 1\}$, and where $\langle i, j\rangle$ denotes nearest neighbours. This can also be expressed in terms of the total energy, $E$, as

$$
Z_{L}(\beta)=\sum_{E} p(E, \beta) e^{-\beta E},
$$

where $p(E, \beta)$ is the density of states. We define the reduced temperature as a dimensionless distance from criticality,

$$
t=1-\frac{\beta}{\beta_{c}}
$$

where $\beta_{c}$ is the critical values of $\beta$. The specific heat of the system, is defined as

$$
C=\frac{1}{V}\left(\left\langle E^{2}\right\rangle_{\beta}-\langle E\rangle_{\beta}^{2}\right),
$$

where $V=L^{3}$ is the volume of a cubic system of linear extent $L$, and $\langle\ldots\rangle_{\beta}$ denotes the thermal average at $\beta$. Close to a continuous phase transition, the specific heat scales with reduced temperature $t$ as

$$
C(t) \simeq A_{ \pm}|t|^{-\alpha}
$$

Table 1: Measurements of the amplitude ratio for the specific heat of the Ising model in three dimensions. The asterisk indicates that Marinari's Monte Carlo estimate was from the impact angle of Fisher zeros.

\begin{tabular}{l|l|l|l}
\hline \hline Authors \& Reference & Year & Method & $A_{+} / A_{-}$ \\
\hline & & & \\
Marinari [16] & 1984 & Monte Carlo* & $0.45(7)$ \\
Belanger and Yoshizawa [17] & 1987 & Experiment & $0.53(1)$ \\
Bagnuls et al [18] & 1987 & Field theory & $0.541(14)$ \\
Liu \& Fisher [19] & 1989 & Series expansions & $0.523(9)$ \\
Hasenbusch \& Pinn [20] & 1998 & Monte Carlo & $0.560(10)$ \\
Nowicki et al [21] & 2001 & Experiment & $0.536(5)$ \\
Campostrini et al [22] & 2002 & Series expansions & $0.532(3)$ \\
Canfora et al [23] & 2009 & Phenomonology & 0.54 \\
Feng \& Blöte [11] & 2010 & Monte Carlo & $0.532(7)$ \\
Hasenbusch [12] & 2010 & Monte Carlo & $0.536(2)$ \\
\hline \hline
\end{tabular}


where the amplitudes $A_{+}$and $A_{-}$correspond to $t>0$ and $t<0$ respectively. The susceptibility is also of interest and can be defined as

$$
\chi=\frac{1}{V}\left(\left\langle M^{2}\right\rangle_{\beta}-\langle M\rangle_{\beta}^{2}\right)
$$

where $M=\sum_{i} \sigma_{i}$ is the magnetisation. One can estimate, for instance, the critical point using the scaling of the specific heat maxima with the lattice size. The currently widely accepted value [24] for this point is $\beta_{c}(L=\infty)=0.2216546$.

A complex zero in the partition function indicates a non-analyticity in the free energy. In the thermodynamic limit $(L \rightarrow \infty)$, the pinching of such zeros of the real temperature axis precipitates a phase transition. Nevertheless we can study the transition in a finite system by analytic continuation to complex temperatures, $\beta=\eta+i \xi$. In this case the partition function includes both oscillating and damping factors:

$$
Z(\beta)=\sum_{E} p(E, \beta) e^{-(\eta+i \xi) E}=\sum_{E} p(E, \beta) e^{-\eta E}[\cos (\xi E)-i \sin (\xi E)] .
$$

Rescaling with $Z[\operatorname{Re}(\beta)]$ we define the quantity:

$$
\begin{aligned}
R(\eta, \xi) & =\frac{Z(\beta)}{Z[\operatorname{Re}(\beta)]}=\frac{\sum_{E} p(E, \beta) e^{-\eta E}[\cos (\xi E)-i \sin (\xi E)]}{\sum_{E} p(E, \beta) e^{-\eta E}} \\
& =\langle\cos (\xi E)\rangle_{\eta}-i\langle\sin (\xi E)\rangle_{\eta} .
\end{aligned}
$$

Therefore the partition function at complex temperature $\beta=\eta+i \xi$ can be constructed using expectation values taken at real temperatures $\beta=\eta$. A Fisher zero is a complex temperature value $(\eta, \xi)$ such that $R=0$. These values can be ordered by their distance from $\beta_{c}(\infty)$ and we denote the first and second zeros by $\beta^{(1)}$ and $\beta^{(2)}$, respectively.

Our objective is to obtain the locations of the Fisher zeros for several system sizes. To achieve this we performed MC simulations in the vicinity of the critical temperature. The crucial point is that we can use histogram reweighting techniques [25] to evaluate $\langle\cos (\xi E)\rangle_{\eta}$ and $\langle\sin (\xi E)\rangle_{\eta}$ near the simulation temperature. With this in hand, we can then minimise $|R|$ using numerical optimisation methods [26].

\section{$3 \quad$ Finite-Size Scaling of the Fisher Zeros}

In Ref.[27] the finite-size scaling (FSS) of the complex thermal coupling (or Fisher) zeros was obtained. The zeros nearest to the real axis $\beta^{(1)}$ scale with lattice size as

$$
\beta^{(1)}=L^{-1 / \nu} f^{-1}(0),
$$

where $f^{-1}(0)$ is a complex number in general. So, both the real and imaginary parts of the Fisher zeros should scale with the same exponent $1 / \nu$. In Appendix A, we discuss an exception of this behavior, and determine under what conditions the real and imaginary parts scale with different power laws. 
In addition there is a relationship between the impact angle of Fisher zeros and the critical amplitudes of the specific heat. If $\phi$ is the angle between the negative sense of the real temperature-parameter axis and the locus of nearby Fisher zeros in the thermodynamic limit, this relationship is

$$
\tan [(2-\alpha) \phi]=\frac{\cos (\pi \alpha)-A_{-} / A_{+}}{\sin (\pi \alpha)}
$$

Notice that if, instead, we define the impact angle $\phi$ as between the positive sense of the real temperature axis and the locus of zeros then we obtain a similar equation with the roles of $A_{-}$and $A_{+}$interchanged:

$$
\tan [(2-\alpha) \phi]=\frac{\cos (\pi \alpha)-A_{+} / A_{-}}{\sin (\pi \alpha)} .
$$

It is clear from either equation that, since the critical exponent $\alpha$ and the amplitude ratio $A_{-} / A_{+}$are universal, the impact angle is also an universal quantity. If $\phi=\pi / 2$, these equations give that $A_{+}=A_{-}$, which is physically reasonable; vertical impact signals symmetry on either side of the phase transition and equality of amplitudes.

In this paper, we will follow the first parametrization of the impact angle: $\phi>0$ will be the angle between the negative direction of the real temperature axis and the Fisher-zeros locus. We will then use the relationship (3.2) to determine the amplitude ratio $A_{-} / A_{+}$in a manner independent to [16], and different from Refs. [11, 12, 17, 18, 19, 20, 21, 22] (see Table 1). We will show that the different methods yield compatible results.

\section{Simulation Details}

We performed extensive simulations of the model for linear lattice sizes from $L=4$ to $L=72$ with periodic boundary conditions. The spins were updated by combining ten Wolff single-cluster algorithms with a Metropolis one. We define in this way our unitary Monte Carlo sweep (MCS). We measured after every MCS, performing $10^{7}$ measures for each lattice size after thermalisation. We used a 64-bits pseudo-random generator combining a Parisi-Rapuano wheel with a congruential one [28].

We simulated twenty independent pseudo-samples for each lattice size starting from random configurations. We merged their MC histories checking that every pseudo-sample was thermalised. To check this point we can plot the logarithmic binning of some quantities for different samples and see the clear compatibility of the plateaus between samples. This is illustrated in Fig. 1 for our largest system, for both the specific heat (2.4) and the magnetic susceptibility (2.6).

We have performed two set of simulations. Firstly we simulated every lattice size at $\beta_{c}(L=\infty)=0.2216546$ 1, as this should be the more direct approach to estimate critical quantities. Nevertheless, we observed unusually large error bars (always computed via jackknife blocking between samples) for the locations of the second zeros in the larger

\footnotetext{
${ }^{1}$ In terms of the associated variable $u=\exp (-4 \beta)$, this is given by $u_{c}=\exp \left(-4 \beta_{c}\right)=0.4120468$.
} 


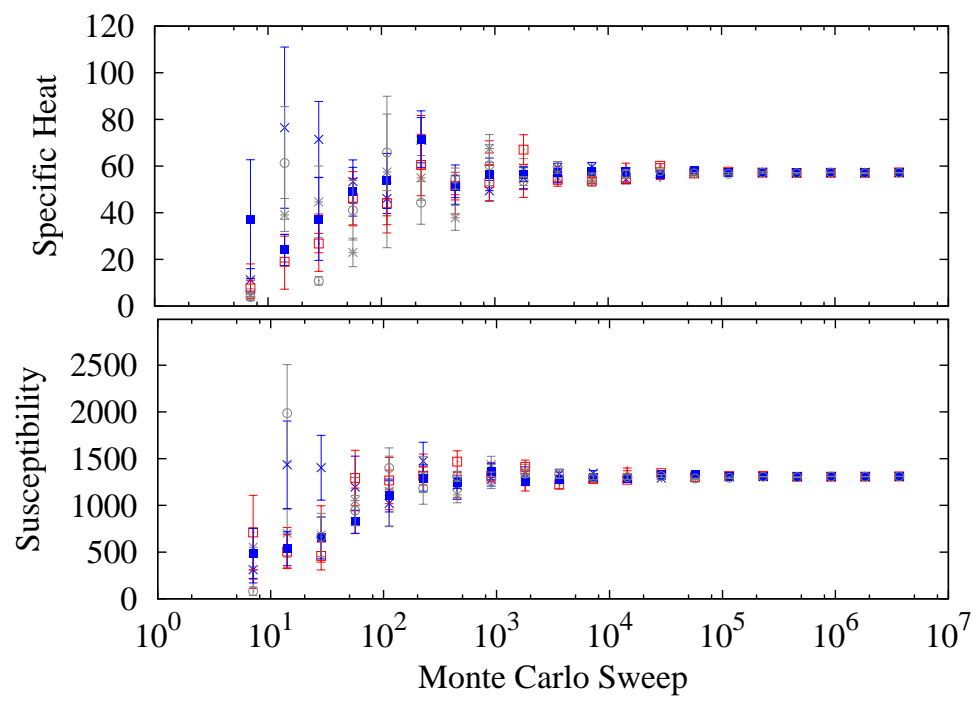

Figure 1: (Color online) Log-binning of susceptibility and specific heat for five random pseudosamples with $L=72$. Error bars are typical deviations inside each bin. The first block only includes seven measurements, explaining the deviations for small times.

lattices. We then performed simulations closer to the previously estimated locations of these second zeros for every $L \geq 24$. We call these new temperature set $\left\{\beta_{\text {sim }}^{\prime}\right\}$. We obtained fully compatible values for the first zero location from these new temperatures

Table 2: Simulation details for each system size $L . N_{\text {th }}$ denotes the number of MCS (in units of $10^{6}$ ) performed in each pseudosample before taking measurements. We simulated every $L$ at $\beta_{c}(\infty)=0.2216546$. In addition, in the third column, we present the second simulation temperature used for the largest lattices, $\beta_{\text {sim }}^{\prime}$.

\begin{tabular}{|r|c|c|}
\hline \hline$L$ & $N_{\text {th }}$ & $\beta_{\text {sim }}^{\prime}$ \\
\hline 4 & 1 & - \\
6 & 1 & - \\
8 & 1 & - \\
12 & 1 & - \\
16 & 1 & - \\
20 & 5 & - \\
24 & 5 & 0.22390 \\
32 & 10 & 0.22300 \\
48 & 10 & 0.22259 \\
56 & 30 & 0.22239 \\
64 & 40 & 0.22213 \\
72 & 50 & 0.22200 \\
\hline \hline
\end{tabular}




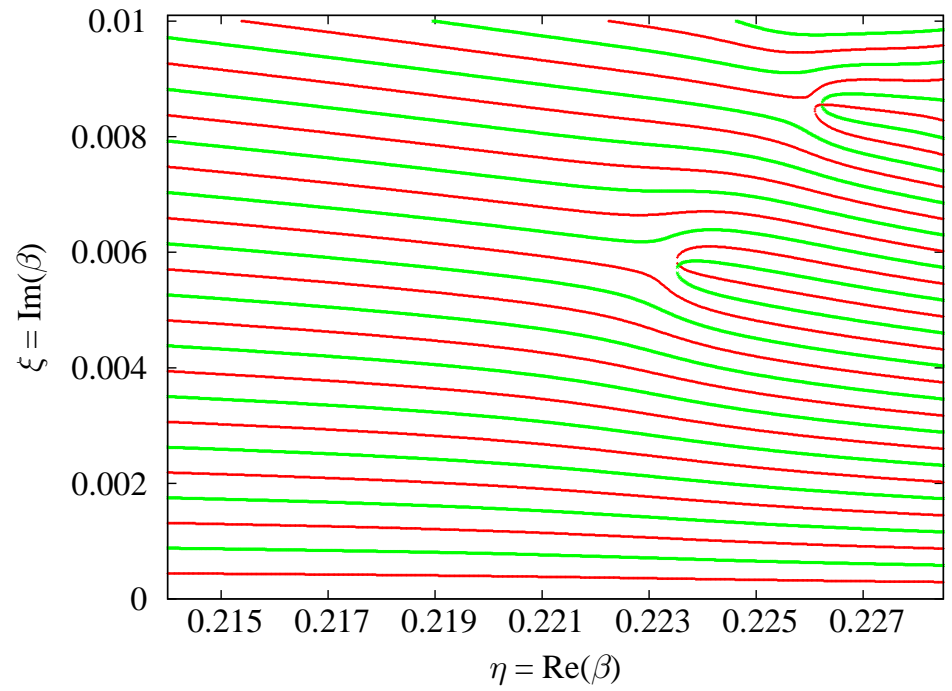

Figure 2: (Color online) Graphical analysis for $L=16$ with $2 \times 10^{7}$ measurements and a $1000 \times 1000$-point extrapolation grid. Dark (red) lines represent the points where $\langle\cos (\xi E)\rangle_{\eta}$ changes sign, light (green) lines are the corresponding signals for $\langle\sin (\xi E)\rangle_{\eta}$. A crossing of the lines is an imaginary zero of the partition function. The extrapolation range allowed the location of the first two zeros.

and we strongly reduced the error bars for the second zeros. It provided us a clear evidence of the sensibility of the second zero location and discouraged us from studying higherindex zeros. In Table 2 we give the concrete simulation information for each system size. The total estimated simulation time is around a year of a $3 \mathrm{GHz} \mathrm{CPU}$.

\section{Results}

To determine the values of the universal amplitude ratios we first obtained the Fisher zeros which lie closest to the real temperature axis for each system size. We used the two-step procedure described in Ref.[26], that is: graphical estimation of the approximate zero location followed by a multidimensional numerical minimization starting from the previously estimated point.

For the graphical estimation we had to perform an extrapolation in $\operatorname{Re}(\beta)=\eta$ (via histogram reweighting, see for example, Ref. [29]) and $\operatorname{Im}(\beta)=\xi$. Our goal is to obtain the points where $\langle\cos (\xi E)\rangle_{\eta}$ and $\langle\sin (\xi E)\rangle_{\eta}$ vanish simultaneously. We defined the secure $\eta$ extrapolation range using the square-root of the energy fluctuations as showed in the reweighting method [29]. For the optimum $\xi$ range we used the known scaling of the imaginary parts of the zeros with the system size. A compromise must be achieved between resolution (number of extrapolated points) and computation time. The analysis time for a lattice with $2 \times 10^{8}$ measures was around a week of a $3 \mathrm{GHz} \mathrm{CPU}$ for a extrapolation grid with $300 \times 300$ points. In Fig. 2 we show the output of one of our analyses. 
Table 3: First and second partition function zeros in terms of $u=\exp (-4 \beta)$, where $\beta$ is the inverse temperature. Notice that if $u$ is a zero its complex conjugate, $u^{*}$, is also.

\begin{tabular}{|r|l|l|l|l|}
\hline \hline$L$ & $\operatorname{Re}\left(u^{(1)}\right)$ & $\operatorname{Im}\left(u^{(1)}\right)$ & $\operatorname{Re}\left(u^{(2)}\right)$ & $\operatorname{Im}\left(u^{(2)}\right)$ \\
\hline 4 & $0.3842660(79)$ & $-0.0877447(77)$ & $0.344594(055)$ & $-0.143306(049)$ \\
6 & $0.3975600(88)$ & $-0.0454084(75)$ & $0.377482(124)$ & $-0.072941(165)$ \\
8 & $0.4027230(57)$ & $-0.0285868(44)$ & $0.390136(159)$ & $-0.045566(171)$ \\
12 & $0.4070150(32)$ & $-0.0149382(25)$ & $0.400370(224)$ & $-0.023291(188)$ \\
16 & $0.4088090(27)$ & $-0.0094326(32)$ & $0.404832(156)$ & $-0.014731(191)$ \\
20 & $0.4097530(30)$ & $-0.0066037(20)$ & $0.406870(211)$ & $-0.010347(227)$ \\
24 & $0.4103170(18)$ & $-0.0049403(18)$ & $0.408153(030)$ & $-0.007684(030)$ \\
32 & $0.4109431(15)$ & $-0.0031260(20)$ & $0.409582(034)$ & $-0.004871(034)$ \\
48 & $0.4114612(09)$ & $-0.0016399(08)$ & $0.410759(037)$ & $-0.002597(038)$ \\
56 & $0.4115855(06)$ & $-0.0012821(10)$ & $0.411005(022)$ & $-0.001995(026)$ \\
64 & $0.4116732(07)$ & $-0.0010367(07)$ & $0.411201(015)$ & $-0.001615(022)$ \\
72 & $0.4117365(07)$ & $-0.0008596(06)$ & $0.411343(016)$ & $-0.001324(014)$ \\
\hline \hline
\end{tabular}

Once we knew the approximate locus of a given zero, we performed an AMOEBA multidimensional minimization [30] starting from this point. In this way we computed the zeros location with quite high accuracy. In Table 3 we display the results for the locations of the first two zeros for each lattice size in terms of the conjugate variable $u=\exp (-4 \beta)$. All error bars are computed defining jackknife blocks between the twenty pseudo-samples. The larger error bars for the estimations of the second zeros for the smallest lattices $(L \leq 20)$ are due to the longer temperature extrapolation, see table 2 , In order to define the impact angle we use the locus with $\operatorname{Im} \beta>0$ or $\operatorname{Im} u<0$.

\subsection{Critical Exponents}

As was stated in Sec $\underline{3}$, we can obtain the critical exponent $\nu$ from the scaling of the real and imaginary part of the zeros (see Appendix). The scaling of the first two zeros is shown on a double-logarithmic scale in Fig. 3. As an additional estimator, we can use the distance from the complex zero to the critical point, $\left|u-u_{c}\right|$. Therefore we fit each of $\operatorname{Im}\left(u^{(j)}\right), u_{c}-\operatorname{Re}\left(u^{(j)}\right)$ and $\left|u^{(j)}-u_{c}\right|$ to the form

$$
a L^{-1 / \nu}\left(1+b L^{-\omega}\right)
$$

which includes the leading correction-to-scaling term. Firstly we tried to obtain both $\nu$ and $\omega$ from fits of our data sets. We were not able to obtain them simultaneously with the same high accuracy as that in Ref.31]. Eliminating $\nu$ by taking ratios and fitting $\operatorname{Im}\left(u^{(j)}\right) /\left|\operatorname{Re}\left(u^{(j)}\right)-u_{c}\right|$ to $A+B L^{-\omega}$ gives the estimate $\omega=0.77(9)$ using $L=12$ to $L=72$ or $\omega=0.63(16)$ using $L=16$ to $L=72$. These compare reasonably to the estimate $\omega=0.832(6)$ in Ref. 31]. Next we decided to focus on the computation of the critical exponent $\nu$. To this end, we fixed the value $\omega=0.832(6)$ [31] and obtained the 
Table 4: Estimated values for the critical exponent $\nu$ from different estimators (first column). The first error bar correspond to the statistical error, while the second one is due to the uncertainty in $\omega$. We give in brackets the size range used in each fit.

\begin{tabular}{|r|c|c|}
\hline \hline & $u^{(1)}$ & $u^{(2)}$ \\
\hline $\operatorname{Re}(u)-u_{c}$ & {$[8-72]: 0.6328(12)(2)$} & {$[4-72]: 0.6576(82)(5)$} \\
$\operatorname{Im}(u)$ & {$[6-72]: 0.63048(25)(7)$} & {$[4-72]: 0.6344(44)(3)$} \\
$\left|u-u_{c}\right|$ & {$[8-72]: 0.63052(41)(2)$} & {$[4-72]: 0.6388(39)(3)$} \\
\hline \hline
\end{tabular}

estimates for $\nu$ shown in Table 4. We discarded data from small sizes until we reached a good value for the confidence level (CL) of the fit 2 To further ensure our control of the corrections to scaling, we checked that our fits are compatible with the fits obtained discarding the next smallest $L$ value. We give the error bar corresponding to these latter fits.

Taking our best estimation (the one with the smaller error bar), we obtain the value 3 :

$$
\nu=0.63048(32) \text {. }
$$

This is in very good agreement with the world mean value provided in Ref. 24] (namely $\nu=0.6301(4)$ ). For comparison, the estimate coming from Ref.[31] is $\nu=0.63002(10)$.

The specific-heat critical exponent can then be estimated using the hyperscaling relation

$$
\nu d=2-\alpha,
$$

where $d=3$ in the present case. We find

$$
\alpha=0.1086(10) .
$$

The world average coming from Ref. [24] is $\alpha=0.1097(12)$ and the estimate from from Ref.[31] is 0.10994(30).

The conjectures regarding exact values in Refs. [1, 2] include a logarithmic divergence in the specific heat. According to the scaling relations for logarithmic corrections, such a logarithm would necessitate the vanishing of $\alpha$ and an impact angle other than $45^{\circ}$ [14. Since the estimate for $\alpha$ in Eq.(5.4) is over 100 standard deviations away from zero, these conjecture are unsupported.

\subsection{Impact Angle and Amplitude Ratios}

We also considered it interesting to contrast the location of the Fisher zeros with the temperatures where the specific heat is maximum. For every lattice size, we extrapolated

\footnotetext{
${ }^{2}$ The CL is the probability that $\chi^{2}$ would be bigger than the observed value, supposing that the statistical model is correct. As a rule, we consider a fit insufficient whenever CL $<10 \%$.

${ }^{3}$ Another test of the robustness of our data is to perform the weighted mean of the data in Table 4 (where the weights are their inverse variances). In this way we obtain $\bar{\nu}=0.63069(29)$ and $\bar{\alpha}=0.1079(9)$.
} 

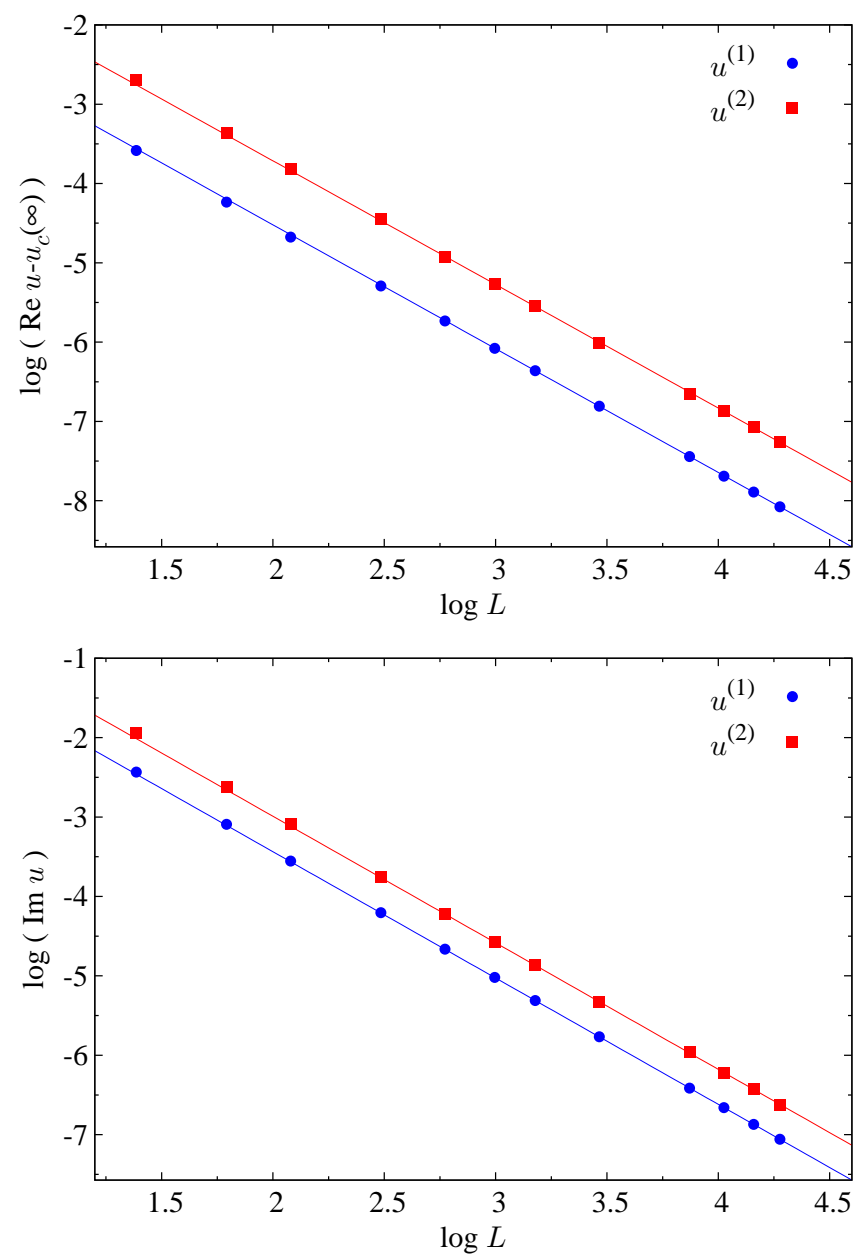

Figure 3: (Color online) Top: Scaling of the distance from $u_{c}(\infty)=0.41204677$ of the real part of the first (blue circles) and second (red squares) Fisher zeros. Bottom: Scaling of the imaginary part of the first (blue circles) and second (red squares) Fisher zeros. In each case the error bars are smaller than the symbols.

Eq. (2.4) from the simulated temperature to obtain its maximum, see Fig. 4. In Fig. 5 we plot the locations of these maxima and the locations of the Fisher zeros for every lattice size. From this plot one can see that the angle between the positive sense of the real axis, the specific-heat pseudocritical point and the first Fisher zero change significantly as the lattice size increases.

There are very few works in the literature which use the impact angle of the Fisher zeros to determine amplitude ratios. Those which do exist are rather old and only had access to very small lattice sizes. Marinari's estimate [16] $A_{+} / A_{-}=0.45(7)$ is based on lattices of extent $L=4$ to $L=8$. An additional motivation for our investigations is to re-examine the impact angle in the light of the much larger lattices available to us today. Unfortunately, there is no FSS theory for the impact angle for Fisher zeros 4 . However,

\footnotetext{
${ }^{4}$ One could attempt to build a FSS theory for the impact angle by noting that, in the infinite-volume
} 


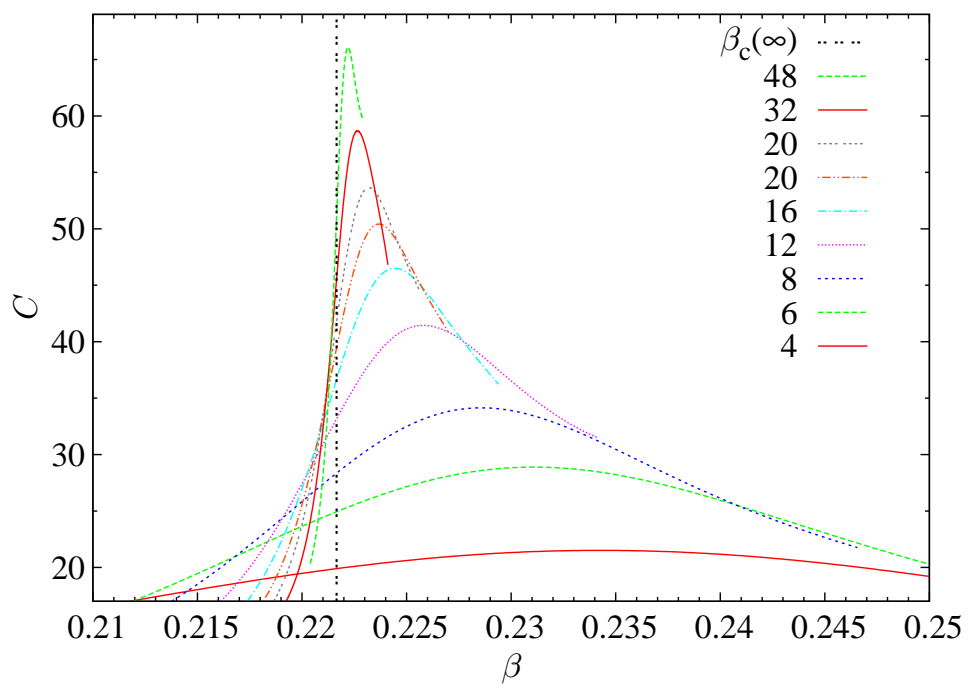

Figure 4: (Color online) Specific heat as a function of the inverse temperature for different system sizes. We can see the clear approach to the asymptotic value $\beta_{c}(\infty)=0.2216546$ as the system size increases.

Table 5: Angles formed between the first and second zeros and the real axis in the $u$ and $\beta$-planes.

\begin{tabular}{|r|r|r|r|r|r|r|}
\hline \hline$L$ & $\phi_{1, c}(u)$ & $\phi_{1, c}(\beta)$ & $\phi_{2, c}(u)$ & $\phi_{2, c}(\beta)$ & $\phi_{1,2}(u)$ & $\phi_{1,2}(\beta)$ \\
\hline 4 & $72.431(5)$ & $78.815(5)$ & $64.79(2)$ & $75.90(2)$ & $54.47(5)$ & $72.15(5)$ \\
6 & $72.31(1)$ & $75.55(1)$ & $64.64(9)$ & $70.1(1)$ & $53.9(2)$ & $62.6(2)$ \\
8 & $71.93(1)$ & $73.96(1)$ & $64.3(2)$ & $67.6(2)$ & $53.4(5)$ & $58.8(5)$ \\
12 & $71.38(1)$ & $72.43(1)$ & $63.3(5)$ & $65.0(5)$ & $51.5(9)$ & $54.2(9)$ \\
16 & $71.05(2)$ & $71.72(2)$ & $63.9(6)$ & $65.0(6)$ & $53(2)$ & $55(2)$ \\
20 & $70.84(2)$ & $71.30(2)$ & $63(1)$ & $64(1)$ & $52(2)$ & $54(2)$ \\
24 & $70.72(2)$ & $71.06(2)$ & $63.1(2)$ & $63.7(2)$ & $51.7(4)$ & $53.6(4)$ \\
32 & $70.54(3)$ & $70.76(3)$ & $63.2(4)$ & $63.5(3)$ & $52.1(8)$ & $52.6(8)$ \\
48 & $70.34(6)$ & $70.45(6)$ & $63.6(7)$ & $63.8(7)$ & $54(2)$ & $54(2)$ \\
56 & $70.38(8)$ & $70.47(9)$ & $62.4(6)$ & $62.6(6)$ & $51(2)$ & $51(2)$ \\
64 & $70.16(6)$ & $70.24(5)$ & $62.4(5)$ & $62.5(5)$ & $51(1)$ & $51(1)$ \\
72 & $70.13(4)$ & $70.19(3)$ & $62.0(6)$ & $62.1(6)$ & $50(2)$ & $50(2)$ \\
\hline \hline
\end{tabular}

a number of ways to estimate the impact angle suggest themselves. These include the limit, one may describe the locus of zeros as $u(r)=u_{c}+r \exp (i \phi(r))$. Taylor expanding $\phi(r)=\phi+r \phi^{\prime}+\ldots$, one finds that the impact angle is given by

$$
\tan \phi=\frac{\operatorname{Im} u(r)}{\operatorname{Re} u(r)-u_{c}}-r \phi^{\prime} \sec ^{2} \phi+\mathcal{O}\left(r^{2}\right) .
$$




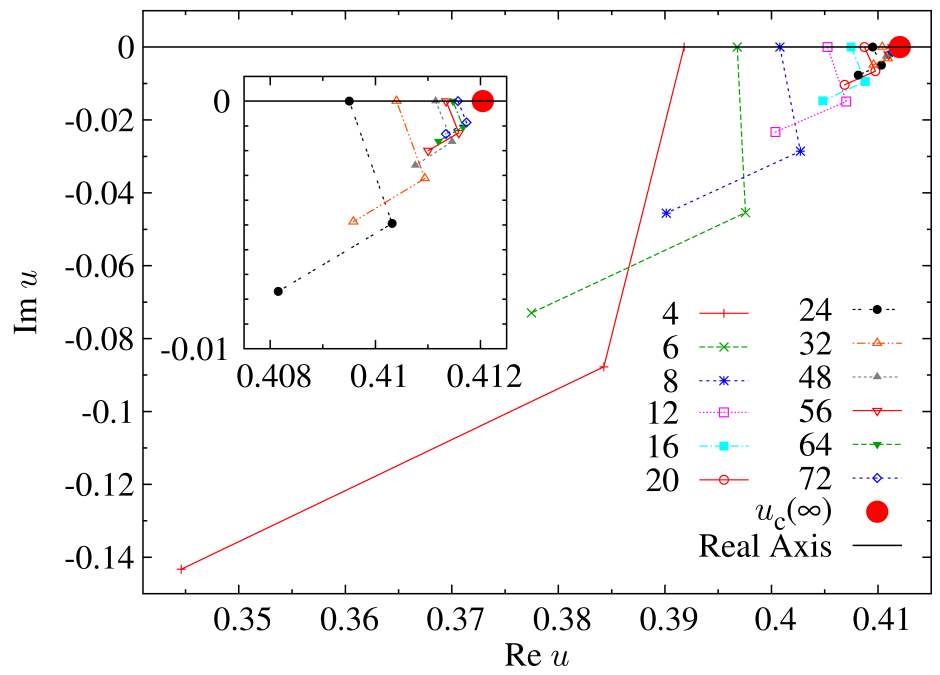

Figure 5: (Color online) First two zeros as a function of the system size. The locations of the pseudocritical temperatures from the maximum of the specific heat are also given on real axis. We indicate with a large red disc the asymptotic value $u_{c}(\infty)$. The inset is a magnification for larger lattices.

angle at the critical point between the negative sense of the real axis and the first or second Fisher zero as well as the angle formed by the impact of the line joining these zeros and the real axis. In Table 5, each of these are listed in both the $u$ - and $\beta$-planes. The notation is as follows:

- $\phi_{j, c}(u)=$ angle at $u_{c}$ between the $j^{\text {th }}$ zero and the real $u$-axis.

- $\phi_{1,2}(u)=$ angle between the line joining $u^{(1)}(L)$ and $u^{(2)}(L)$ and the real $u$-axis.

- $\phi_{j, c}(\beta)=$ angle at $\beta_{c}$ between the $j^{\text {th }}$ zero and the real $\beta$-axis.

- $\phi_{1,2}(\beta)=$ angle between the line joining $\beta^{(1)}(L)$ and $\beta^{(2)}(L)$ and the real $\beta$-axis.

Angles measured in the $u$ - and $\beta$ - planes should converge since the transformation from $\beta$ to $u$ is a conformal one, which preserves angles locally. However, for small lattices, where the zeros are relatively far apart, we may expect different impact angles for the $\beta$ - and $u$-planes. But in the large $L$ region and when the zeros are sufficiently close to the real axis, the conformality of the transformation implies that both angles should be nearly equal. This fact serves as a check that we are approaching the asymptotic region in the

To achieve the known FSS of the zeros $u^{(j)}(L)$ [27, write $r_{j}(L)=R\left(j / L^{d}\right)^{\frac{1}{\nu d}}$. Replacing $r$ by $r_{j}(L)$,

$$
\tan \phi=\tan \phi_{j}(L)-\rho_{j} L^{-\frac{1}{\nu}}+\ldots,
$$

where $\phi_{j}(L)=\operatorname{Im} u^{(j)}(L) /\left(\operatorname{Re} u^{(j)}(L)-u_{c}\right)$ and $u^{(j)}(L) \equiv u\left(r_{j}(L)\right)$ and $\rho_{j}=R j^{1 / \nu d} \phi^{\prime} \sec ^{2} \phi$. Unfortunately we have found this approach fails due to strong corrections. 
computation of the impact angle and we can safely discard the results from lattices that are too small to achieve approximate conformality. We find that approximate conformality kicks in at around $L \gtrsim 32$. This means that the earlier measurements of Refs. [16, 27] are unreliable and justifies our re-visitation of the problem. The results for the various angles and the various lattice sizes are given in Table 5. The angles were obtained for the data simulated at $\left\{\beta_{\text {sim }}^{\prime}\right\}$ to use the correlations between the two zeros to reduce the errors via the jackknife method.

In the absence of a FSS theory for impact angles, we conservatively take the wedge formed by the smallest and largest angles for which the lattices exhibit approximate conformality to approximate the approach of the zeros to the real axis. This wedge can be used to estimate upper and lower bounds for the impact of the locus of Fisher zeros onto the real axis. The impact angle may then be approximated by the average of these bounds, with their difference delivering an error estimate. In Table 6, these averages are listed for lattice sizes that exhibit the desired approximate conformality.

From the values listed in Table 6, we observe a drift towards smaller values of $\phi$ as the lattice size increases. The values for the largest lattice studied $(L=72)$ and its associated estimate for the amplitude ratio are

$$
\phi=59.9(8)^{\circ}, \quad \frac{A_{+}}{A_{-}}=0.58(2) .
$$

On may alternatively attempt to extrapolate to the thermodynamic limit using a correction ansatz5

$$
\phi(L)=\phi+b L^{-\omega},
$$

where $\phi(L)$ is the impact angle computed with the data from the lattice size $L$ and $\phi$ is the asymptotic value of this angle.

With $\omega=0.832(6)$ from (5.2), we obtain (fitting only $L \geq 48$ )

$$
\phi=59.2(1.0)^{\circ}
$$

The corresponding estimate for the amplitude ratio is

$$
\frac{A+}{A_{-}}=0.56(3)
$$

This compares with Hasenbusch's estimate $A_{+} / A_{-}=0.536(2)$ from Ref.[12] (see also Table 11).

\footnotetext{
${ }^{5}$ The rationale for this fit is the following. We know that

$$
\tan \phi(L)=\left(\operatorname{Im}\left(u^{(2)}\right)-\operatorname{Im}\left(u^{(1)}\right)\right) /\left(\operatorname{Re}\left(u^{(2)}\right)-\operatorname{Re}\left(u^{(1)}\right)\right) .
$$

The scaling of the numerator and denominator is given by eq. (5.1), and finally assuming small angles

$$
\phi=\phi(L)+O\left(L^{-\omega}\right) .
$$
}
we obtain 


\section{Conclusions}

Because of recent claims around the values of the critical exponents of the three-dimensional Ising model, and in the light of renewed interest in the measurements of amplitude ratios in this model, we decided to investigate this well-studied model from an alternative perspective, namely that of complex-temperature zeros. In particular, the manner in which such zeros impact onto the real axis dictates the amplitude ratios for the specific heat. Only very old (almost 3 decades old) measurements of this impact angle exist in the literature, so another motivation was to investigate this using the powerful algorithms and large lattices currently at our disposal. Indeed, we have found that previous measurements of the impact angle were made on lattices which were too small to manifest conformality in the complex temperature plane.

In the absence of a finite-size scaling theory for the impact angle, we were able to estimate upper and lower bounds for its value. These lead to an estimate $\phi=59.2(1.0)^{\circ}$, which in turn leads to an estimate for the amplitude ratio of $A_{+} / A_{-} 0.56(3)$. In addition to the amplitude ratios, we investigated the correlation-length critical exponent and determined that $\nu=0.63048(32)$. The corresponding value for the specific-heat critical exponent is $\alpha=0.1086(10)$, from hyperscaling. These values are compatible with the most accurate recent measurements, and therefore reinforce confidence in our estimates for the impact angle. All of our estimates are consistent with the values currently widely accepted and are incompatible with the values recently claimed in Refs. [1, 2].

Acknowledgements: RK acknowleges support from EU Programme FP7-People-2010IRSES (Project No 269139), and the ARF Scheme of Coventry University. AGG and JJR acknowlege support from Research Contracts No. FIS2007-60977 (MICINN), GR10158 (Junta de Extremadura) and ACCVII-08 (UEX).

Table 6: Impact angle estimation from the average between $\phi_{1, c}(u)$ and $\phi_{1,2}(u)$ only for the sizes respecting conformality. We also estimate the universal amplitude ratio $A_{+} / A_{-}$ from this angle.

\begin{tabular}{|r|c|c|}
\hline \hline$L$ & Angle Average & $\left(A_{+} / A_{-}\right)_{\text {average }}$ \\
\hline 32 & $61.3(4)$ & $0.610(9)$ \\
48 & $62(1)$ & $0.63(2)$ \\
56 & $60.5(8)$ & $0.59(2)$ \\
64 & $60.5(7)$ & $0.59(2)$ \\
72 & $59.9(8)$ & $0.58(2)$ \\
\hline \hline
\end{tabular}




\section{A Appendix}

In FSS theory, the shift exponent $\lambda$ characterizes the scaling of the pseudocritical point towards the critical point as the thermodynamic limit is approached. The pseudocritical point is given by the location of the specific-heat maximum or by the real part of the first Fisher zero and the approach is as $L^{-\lambda}$. In most models exhibiting higher-order phase transitions, the shift exponent $\lambda$ coincides with the inverse of the correlation-length critical exponent $1 / \nu$, but this is not a direct conclusion of FSS theory and is not always true.

For example, for the Ising model in two dimensions, Ferdinand and Fisher determined that the specific-heat pseudocritical point scales with $\lambda=1 / \nu=1$ 32. However, Ising models defined on other two-dimensional lattices with different topologies have shift exponents which differ from the inverse correlation length critical exponent (see Ref. [33] for a discussion and references therein). This is despite the fact that the critical properties on such lattices are the same as for the torus in the thermodynamic limit. Here we ask the question: Is there a criterion for $\lambda \neq 1 / \nu$ ? We find that there is such a criterion - the specific-heat amplitude ratio must be 1 .

Although a detailed FSS theory for the impact angle is lacking, for sufficiently large $L$ one may expect that $\phi$ is approximated by the angle subtended by the first zero on the real axis at the critical point,

$$
\tan \phi \approx \frac{\operatorname{Im}\left[u^{(1)}(L)\right]}{\operatorname{Re}\left[u^{(1)}(L)\right]-u_{c}} \propto \frac{L^{-1 / \nu}}{L^{-\lambda}} \sim C L^{\lambda-1 / \nu}+\ldots
$$

To find the angle $\phi$, let $L \rightarrow \infty$. If, as in most cases, $\lambda=1 / \nu$, then $\tan \phi=C$, some constant value. If $\lambda<1 / \nu$ then $\tan \phi=0$. But this is impossible as $\lambda$ cannot be less than $1 / \nu$ (otherwise $\nu$ becomes $1 / \lambda$ ).

The only possibility to have $\lambda \neq 1 / \nu$, then, is for $\lambda>1 / \nu$. In this case $\tan \phi$ diverges as $L \rightarrow \infty$, so that $\phi=\pi / 2$. From (3.2), then, one finds $A_{+}=A_{-}$. This is precisely what happens in the Ising and Potts models in $d=2$, where the coincidence of the specific-heat amplitudes is guaranteed by self-duality.

Note added in proof: After submission of this work, we became aware of a recent extended

scaling approach by Campbell and Lundow [34] which yields a universal amplitude ratio $A_{+} / A_{-}=0.540(4)$. This value is also compatible with the estimate presented herein.

\section{References}

[1] J. Kaupuzs, Annalen Phys. 10 (2001) 299.

[2] Z.D. Zhang, Phil. Mag. 87 (2007) 5309.

[3] J. Kaupuzs, arXiv:1103.0888. 
[4] D.J. Klein and N.H. March, Phys. Lett. A 372 (2008) 5052.

[5] J. Strečka, J. Dely and L. Čanová, Physica A 388 (2009) 2394.

[6] F.Y. Yu, B.M. McCoy, M.E. Fisher and L. Chayes, Phil. Mag. 88 (2008) 3093; Z.D. Zhang, Phil. Mag. 88 (2008) 3097; F.Y. Yu, B.M. McCoy, M.E. Fisher and L. Chayes, Phil. Mag. 88 (2008) 3103.

[7] J.H.H. Perk, Phil. Mag. 89 (2009) 761; Phil. Mag. 89 (2009) 769; Z.D. Zhang, Phil. Mag. 89 (2009) 765.

[8] N.H. March and Z.D. Zhang, Phys. Lett. A 373 (2009) 2075.

[9] N.H. March and Z.D. Zhang, J. Math. Chem. 47 (2010) 520.

[10] J. Ławrynowicz, S. Marchiafava and A. Niemczynowicz, Adv. Appl. Clifford Alg. 20 (2010) 733.

[11] X. Feng and H.W.Js. Blöte, Phys. Rev. E 81 (2010) 031103.

[12] M. Hasenbusch, Phys. Rev. B 82 (2010) 174434.

[13] A. Rosengren, J. Phys. A: Math. Gen. 19 (1986) 1709.

[14] R. Kenna, D.A. Johnston and W. Janke, Phys. Rev. Lett. 96 (2006) 115701; Phys. Rev. Lett. 97 (2006) 155702; Erratum, ibid 169901.

[15] N.Sh. Izmailian and Chin-Kun Hu, Phys. Rev. Lett. 86 (2001) 5160; Nucl. Phys. B 808 (2009) 613; N.Sh. Izmailian and Yeong-Nan Yeh, Nucl. Phys. B 814 (2009) 573.

[16] E. Marinari, Nucl. Phys. B 235 (1984) 123.

[17] D.P. Belanger and H. Yoshizawa, Phys. Rev. B 35 (1987) 4823.

[18] C. Bagnuls, C. Bervillier, D.I. Meiron and B.G. Nickel, Phys. Rev. B 35 (1987) 3585.

[19] A.J. Liu and M.E. Fisher, Physica A 156 (1989) 35.

[20] M. Hasenbusch and M. Pinn, J. Phys. A 31 (1998) 6157.

[21] A.W. Nowicki, M. Ghosh, S.M. McClellan, and D.T. Jacobs, J. Chem. Phys. 114 (2001) 4625.

[22] M. Campostrini, A. Pelissetto, P. Rossi and E. Vicari, Phys. Rev. E 65 (2002) 066127.

[23] F. Canfora, Phys. Lett. B 646 (2007) 54-61. M. Astorino, F. Canfora, C. Martinez and L. Parisi, Phys Lett B 664 (2008) 139. M. Astorino, F. Canfora and G. Giribet, Phys. Lett. B 671 (2009) 291.

[24] A. Pelissetto and E. Vicari, Phys. Rept. 368 (2002) 549 . 
[25] M. Falcioni, E. Marinari, M. L. Paciello, G. Parisi, and B. Taglienti, Phys. Lett. B 108 (1982) 331. A. M. Ferrenberg and R. H. Swendsen, Phys. Rev. Lett. 61 (1988) 2635.

[26] N. A. Alves, J. R. Drugowich de Felicio and U. H. E. Hansmann, Int. J. Mod. Phys. C 8 (1997) 1063.

[27] C. Itzykson, R. B. Pearson and J. B. Zuber, Nucl. Phys. B 220 (1983) 415.

[28] G. Parisi and F. Rapuano, Phys. Lett. B 157 (1985) 301.

[29] R. H. Ferrenberg and R. H. Swendsen. Phys. Rev. Lett. 61 (1988) 2635.

[30] W. H. Press, S. A. Teukolsky, W. T. Vetterling and B. P. Flannery, Numerical Recipes: The Art of Scientific Computing (Cambridge University Press, 2007).

[31] M. Hasenbusch, Phys. Rev. B 82 (2010) 174433.

[32] A. E. Ferdinand and M. E. Fisher, Phys. Rev. 185 (1969) 832.

[33] W. Janke and R. Kenna, Phys. Rev. B 65 (2002) 064110.

[34] I.A. Campbell and P.H. Lundow, Phys. Rev. B 83 (2011) 014411. 